\title{
CORRIGENDUM
}

\section{Trends in overweight and obesity and changes in the distribution of body mass index in schoolchildren of Jena, East Germany}

K Kromeyer-Hauschild and K Zellner

European Journal of Clinical Nutrition (2007) 61, 690. doi:10.1038/sj.ejcn.1602729

Correction to: European Journal of Clinical Nutrition (2007) 61, 404-411. doi:10.1038/sj.ejcn.1602533

Since the publication of this issue, the authors have identified errors to Table 2. The table is printed correctly below.
The authors would like to aplogise for any inconvenience caused.

Table 2 Comparative prevalence of overweight and obesity among boys and girls in Jena

\begin{tabular}{|c|c|c|c|c|c|c|c|c|}
\hline & \multicolumn{2}{|r|}{1975} & \multicolumn{2}{|r|}{1985} & \multicolumn{2}{|r|}{1995} & \multicolumn{2}{|r|}{2001} \\
\hline & $\mathrm{N}$ & $\%(95 \% \mathrm{Cl})$ & $\mathrm{N}$ & $\%(95 \% \mathrm{Cl})$ & $\mathrm{N}$ & $\%(95 \% \mathrm{Cl})$ & $\mathrm{N}$ & $\%(95 \% \mathrm{Cl})$ \\
\hline \multicolumn{9}{|l|}{ Overweight } \\
\hline \multicolumn{9}{|l|}{ Boys } \\
\hline German Reference & 40 & $4.0(2.9-5.4)$ & 29 & $3.7(2.5-5.3)$ & 69 & $7.0(5.5-8.7)$ & 86 & $8.9(7.4-10.9)$ \\
\hline International Reference & 60 & $6.0(4.6-7.7)$ & 56 & $7.2(5.5-9.3)$ & 94 & $9.5(7.8-11.5)$ & 128 & $13.3(11.2-15.6)$ \\
\hline \multicolumn{9}{|l|}{ Girls } \\
\hline German Reference & 33 & $3.3(2.4-4.6)$ & 22 & $2.9(2.0-4.2)$ & 64 & $7.0(5.5-8.8)$ & 78 & $8.2(6.8-10.2)$ \\
\hline International Reference & 52 & $5.2(4.0-6.8)$ & 49 & $6.5(4.9-8.6)$ & 104 & $11.4(9.5-13.7)$ & 115 & $12.1(10.1-14.4)$ \\
\hline \multicolumn{9}{|l|}{ Obesity } \\
\hline \multicolumn{9}{|l|}{ Boys } \\
\hline German Reference & 9 & $0.9(0.4-1.7)$ & 6 & $0.8(0.2-1.7)$ & 24 & $2.4(1.7-3.3)$ & 22 & $2.3(1.4-3.4)$ \\
\hline International Reference & 7 & $0.7(0.3-1.4)$ & 5 & $0.6(0.2-1.5)$ & 19 & $1.9(1.2-3.0)$ & 17 & $1.8(1.0-2.8)$ \\
\hline \multicolumn{9}{|l|}{ Girls } \\
\hline German Reference & 8 & $0.8(0.4-1.6)$ & 4 & $0.5(0.2-1.4)$ & 7 & $0.8(0.3-1.6)$ & 17 & $1.8(1.0-2.9)$ \\
\hline International Reference & 7 & $0.7(0.3-1.4)$ & 2 & $0.3(0.03-1.0)$ & 6 & $0.7(0.2-1.5)$ & 9 & $0.9(0.4-1.8)$ \\
\hline
\end{tabular}

$95 \% \mathrm{Cl}=95 \%$ exact confidence limits. 